\title{
Comparative Study to Evaluate Efficacy, Safety and Quality of Life of Metoprolol and Telmisartan versus Metoprolol and Ramipril in Patients of Hypertension
}

\author{
Sonia Arora ${ }^{*}$, Vijay K. Sehgal ${ }^{2}$, Jasbir Singh ${ }^{3}$ and Harcharan Singh ${ }^{4}$ \\ 'Post Graduate Student, \\ 2Professor, \\ ${ }^{3}$ Associate Professor, Department of Pharmacology, Government Medical College, Patiala, \\ Punjab-147001, India; soniadr58@gmail.com \\ ${ }^{4}$ Associate Professor, Department of Medicine, Government Medical College, Patiala, Punjab - 147001, India
}

\begin{abstract}
Background: Hypertension(HTN) is a major cardiovascular disease and is a major worldwide clinical problem. The prevalence of hypertension increases in urban and rural areas. The treatment of hypertension began in the 1960s with oral diuretics. The other modalities of treatment of hypertension are beta - blockers, calcium-channel blockers, alphareceptors blockers, ACE inhibitors and ARBs. The better compliance occurs with single-pill combination, and may be even double or even triple pill combination therapy should be used. Also quality of life was improved better with Metoprolol and Telmisartan as compared with Metoprolol and Ramipril. Quality of life was assessed by SF -36 Quiestionnare. Objective: To compare the effect of Metoprolol and Telmisartan versus Metoprolol and Ramipril on BP and quality of life in patients of hypertension. Material and Methods: In this prospective, open, randomized, parallel group, comparative study, 80 patients of hypertension attending the Cardiology Outpatient Department, Govt. Medical College \& Rajindra Hospital, Patiala were recruited. This randomized comparative study was done on 80 patients for 4 months. Quality of Life: In my project of Quality of life, I had taken total 80 patients and the patients were divided into two groups and 40 patients each of Metoprolol and Ramipril versus Metoprolol and Telmisartan. To assess quality of life questionnaire SF-36 was administered to the patients. Results: There was a marked decrease in SBP and DBP with the use of Metoprolol and Telmisartan than Metoprolol and Ramipril. There was also no change in demographic parameters. There was significant improvement in the quality of life with Metoprolol and Telmisartan. Conclusion: Metoprolol and Telmisartan was a better choice than Metoprolol and Ramipril in treating hypertension as this combination causes more reduction in BP and little effect on HR.
\end{abstract}

Keywords: DBP - Diastolic Blood Pressure, HR - Heart Rate, HRQOL - Health Related Quality of Life, HTN - Hypertension, SBP - Systolic Blood Pressure

\section{Introduction}

Hypertension is a risk factor for cardiovascular diseases. ${ }^{[1]}$ The treatment of hypertension significantly reduces the cardiovascular morbidity and mortality. ${ }^{[2]}$ According to JNC - VII guidelines hypertension is classified as: Normal-<- 120 and $<-80$ (mm of $\mathrm{Hg})$, Pre - hypertensive - 120-139 and 80-89 (mm of Hg), Stage I - 140-159 and 90-99 ( $\mathrm{mm}$ of $\mathrm{Hg}$ ) and Stage II- >-160 and >-100(mm of Hg) ${ }^{[3]}$ Cardiovascular diseases are going to be double by $2020{ }^{[4]}$ Hypertension is responsible for stroke deaths and cardiovascular diseases in India. ${ }^{[4]}$ Thus it is very important to control BP in hypertensive patients. The drugs most commonly used are Beta adrenergic blockers out of which Metoprolol is the most commonly used. ${ }^{[5]}$ Other drugs used are Calcium channel blockers, Angiotensin converting enzyme inhibitors, alpha receptor antagonists and Angiotensin receptor blockers. However according to JNC - VIII guidelines Beta-blockers are not used as initial treatment and treatment is given according to ethnicity. ${ }^{[6-8]}$ The JNC - VIII guidelines recommend that 
the general non-black population initial pharmacologic therapy should include a thiazide-type diuretics, calcium channel blockers, angiotensin converting enzyme inhibitors and angiotensin receptor blockers whereas in general black population initial therapy should include a thiazide type diuretics or calcium channel blockers. This difference is based on the fact that the black patients have a smaller reduction in blood pressure when given ACEI or ARBs. ${ }^{[8]}$ Another popularly used drug among the antihypertensives is Telmisartan which is used in my study along with Ramipril separately in combination with Metoprolol. Angiotensin receptor blockers were developed because of their good efficacy and lower side effect profile than angiotensin converting enzyme inhibitors. ${ }^{[9]}$ So, the aim of the study was to evaluate antihypertensive efficacy and effect on quality of life on patients of hypertension.

\section{Material and Methods}

In the prospective, open, randomized, parallel group, comparative study, 80 patients of HTN were included. The study was conducted for 4 months and follow up was done at $2^{\text {nd }}$ and $4^{\text {th }}$ months. A written informed consent was taken from patients after explaining them about study drugs. A thorough history such as HTN, bronchial asthma, tuberculosis, smoker and alcoholic was taken. Patients were randomly divided into two groups.

Group 1 patients were started on Metoprolol and Ramipril at a dose of $50 \mathrm{mg}$ and $2.5 \mathrm{mg}$ respectively. The subsequent titrations were carried up to maximum recommended dose of $200 \mathrm{mg}$ with Metoprolol and $20 \mathrm{mg}$ with Ramipril depending on therapeutic response.

Group 2 patients were put on Metoprololand Telmisartan at a dose of $50 \mathrm{mg}$ and $20 \mathrm{mg}$ respectively. The subsequent titrations were carried up to maximum recommended dose of $200 \mathrm{mg}$ with Metoprolol and $80 \mathrm{mg}$ with Telmisartan.

\section{Quality of Life}

In my project of Quality of life, I had taken total 80 patients who were prescribed Metoprolol and Ramipril and Metoprolol and Telmisartan. The patients were randomly given medications of which two groups were made and 40 patients each of Metoprolol and Ramipril versus Metoprolol and Telmisartan.

To assess quality of life questionnaire SF-36 was administered to the patients. The questionnaire was administered face to face to every patient and it took about $15 \mathrm{~min}$ to administer this questionnaire to the patient.

$\mathrm{SF} 36 \mathrm{v} 2$ is a multidimensional questionnaire. It is composed of 36 items, and it covers eight domains of health: physical functioning (10 items), role limitations caused by physical health problems (4 items), pain ( 2 items), general health perceptions (5 items), energy and/or fatigue (4 items), social functioning ( 2 items), role limitations caused by emotional health problems ( 3 items), and emotional well-being (5 items). Each question in the SF-36 is given a score and it is later translated to a scale number.

Composition of domains of SF-36 Questionnaire

\begin{tabular}{|l|l|}
\hline Domains & Questions \\
\hline 1. Physical functioning & Q 3 a, b, c, d, e, f, g, h, i, j \\
\hline $\begin{array}{l}\text { 2. Role limitations due to } \\
\text { physical health }\end{array}$ & Q 4 a, b, c, d \\
\hline $\begin{array}{l}\text { 3. Role limitations due to } \\
\text { emotional health }\end{array}$ & Q 5 a, b, c \\
\hline 4. Fatigue/Vitality & Q 9a, 9e, 9g, 9i \\
\hline 5. Emotional well -being & Q 9b, 9c, 9d, 9f, 9h \\
\hline 6. Social functioning & Q 6, Q10 \\
\hline 7. Pain & Q 7, Q8 \\
\hline 8. General health & Q 1, Q2, Q11a, b, c, d \\
\hline
\end{tabular}

The responses were recorded on SF-36 Questionnaire as told by the patients. Each question in the SF-36 was given a score and it is later translated to a scale number. The responses with scale number were translated from 0 to 100 . The responses with 0 were given the worst score and responses with score 100 were given the highest score.

\begin{tabular}{|l|l|l|}
\hline Question & Original response & Scoring \\
\hline & 1 & 100 \\
$1,2,6,8$ & 2 & 75 \\
& 3 & 50 \\
& 4 & 25 \\
\hline $3 a, 3 b, 3 c, 3 d, 3 e, 3 f, 3 g, 3 h$, & 1 & 0 \\
$3 \mathrm{i}, 3 \mathrm{j}$ & 2 & 0 \\
& 5 & 50 \\
$4 \mathrm{a}, 4 \mathrm{~b}, 4 \mathrm{c}, 4 \mathrm{~d}, 5 \mathrm{a}, 5 \mathrm{~b}, 5 \mathrm{c}$ & 1 & 100 \\
\hline
\end{tabular}




\begin{tabular}{|l|l|l|}
\hline & 1 & 0 \\
7 & 2 & 20 \\
& 3 & 40 \\
& 4 & 60 \\
& 5 & 80 \\
9a,9b,9c,9d,9e,9f,9g,9h,9i & 6 & 100 \\
\hline & 1 & 100 \\
& 2 & 80 \\
& 3 & 60 \\
$10,11 a, 11 b, 11 c, 11 d$ & 5 & 40 \\
& 6 & 20 \\
& 1 & 0 \\
\hline & 2 & 100 \\
& 3 & 75 \\
& 4 & 50 \\
& 5 & 25 \\
\hline
\end{tabular}

\section{Study Design}

In this prospective, open, randomized, parallel group, comparative study, 80 patients of hypertension attending the Cardiology Outpatient Department, Govt. Medical College and Rajindra Hospital, Patiala were recruited. Patients were selected based on the following criteria:-

Inclusion Criteria:

- Patients with newly diagnosed HTN.
- Those patients who have discontinued antihypertensive medication.

\section{Exclusion Criteria:}

- Patients with history of hypersensitivity to Metoprolol, Telmisartan and Ramipril.

- Pregnant/lactating/women planning to conceive.

- Patients on other anti-hypertensive therapy.

- Patients of secondary hypertension.

- Patients with impaired liver function.

- Patients with impaired kidney function.

- Patients with bronchial asthma, chronic pulmonary disease and peripheral arterial disease.

\section{Statistical Analysis}

Statistical analysis was done using IBM SPSS version 22 software. $p$-value $<0.05$ was considered significant.

Baseline characteristics of patients with Metoprolol and Telmisartan versus Metoprolol and Ramipril on BP were summarized in Table 1. There was no significant difference in $\mathrm{BP}$ and other demographic parameters at baseline but at $2^{\text {nd }}$ month there was a significant difference in $\mathrm{BP}$ and at $4^{\text {th }}$ month there was a highly significant difference in $\mathrm{BP}$ at supine position.

Table 1. Baseline characteristics of Group 1 and Group 2

\begin{tabular}{|l|l|l|}
\hline Characteristics & Group 1 & Group 2 \\
\hline Number of patients & 40 & 40 \\
\hline Age Range(years) & $45-79$ & $40-83$ \\
Mean Age (years) & $61.90 \pm 8.58$ & $61.18 \pm 9.26$ \\
\hline Sex (Male / Female) & $25 / 15$ & $14 / 26$ \\
\hline Systolic BP (mm Hg) & $141.25 \pm 9.43$ & $144.30 \pm 9.35$ \\
\hline Diastolic BP (mm Hg) & $91.80 \pm 8.91$ & $95.20 \pm 8.54$ \\
\hline Heart Rate (Pulse/minute) & $77.42 \pm 11.97$ & $74.75 \pm 9.66$ \\
\hline Hb & $11.04 \pm 0.85$ & $10.78 \pm 1.00$ \\
\hline FBS & $87.85 \pm 16.61$ & $86.75 \pm 13.59$ \\
\hline Blood urea & $30.95 \pm 2.60$ & $31.35 \pm 2.91$ \\
\hline Serum Creatinine & $0.99 \pm 0.15$ & $1.04 \pm 0.21$ \\
\hline Lipid profile & $162.50 \pm 13.21$ & $161.15 \pm 12.13$ \\
\hline SGOT & $22.25 \pm 4.73$ & $22.60 \pm 6.20$ \\
\hline SGPT & $23.70 \pm 4.88$ & $24.12 \pm 5.24$ \\
\hline
\end{tabular}

Comparison of Systolic BP and Diastolic BP at Baseline, $2^{\text {nd }}$ month and at $4^{\text {th }}$ month in both the groups is mentioned in Table 2. Table 3 describes the comparison of HR of two groups at different visits. Quality of life parameters within Metoprolol and Ramipril within group 1 are described in Table 4 and Quality of life parameters within Metoprolol and Telmisartan within group 2 are described in Table 5. Comparison of Quality of life parameter between 2 groups at baseline is illustrated in Table 6. Table 7 describes the comparison of Quality of life between 2 groups at $4^{\text {th }}$ month. 
Table 2. Comparison of Systolic BP and Diastolic BP at baseline, $2^{\text {nd }}$ month and at $4^{\text {th }}$ month in both the groups

\begin{tabular}{|l|l|l|l|l|l|l|}
\hline Time Interval & $\begin{array}{l}\text { Group1 } \\
\text { Mean } \pm \text { S.D. }\end{array}$ & $\begin{array}{l}\text { Group 2 } \\
\text { Mean } \pm \text { S.D. }\end{array}$ & $\begin{array}{l}\text { Mean } \\
\text { Diff. }\end{array}$ & T & P value & Sig. \\
\hline $\begin{array}{l}\text { Baseline } \\
\text { SBP }\end{array}$ & $141.25 \pm 9.43$ & $144.30 \pm 9.35$ & $3.05 \pm 0.08$ & 1.452 & 0.150 & NS \\
DBP & $91.80 \pm 8.91$ & $95.20 \pm 8.54$ & $3.40 \pm 0.37$ & 1.742 & 0.085 & NS \\
\hline $\begin{array}{l}2 \text { Months } \\
\text { SBP }\end{array}$ & $132.65 \pm 9.46$ & $136.82 \pm 8.85$ & $4.18 \pm 0.61$ & 2.038 & 0.045 & S \\
DBP & $84.07 \pm 7.61$ & $87.92 \pm 6.58$ & $3.85 \pm 1.03$ & 2.420 & 0.018 & S \\
\hline $\begin{array}{l}4 \text { Months } \\
\text { SBP }\end{array}$ & $127.40 \pm 9.14$ & $120.85 \pm 8.23$ & $6.55 \pm 0.91$ & 3.369 & 0.001 & HS \\
DBP & $81.05 \pm 6.21$ & $85.75 \pm 4.60$ & $4.70 \pm 1.61$ & 3.846 & 0.001 & HS \\
\hline
\end{tabular}

Bar Diagram Showing SBP in 2 Groups at Different Visits

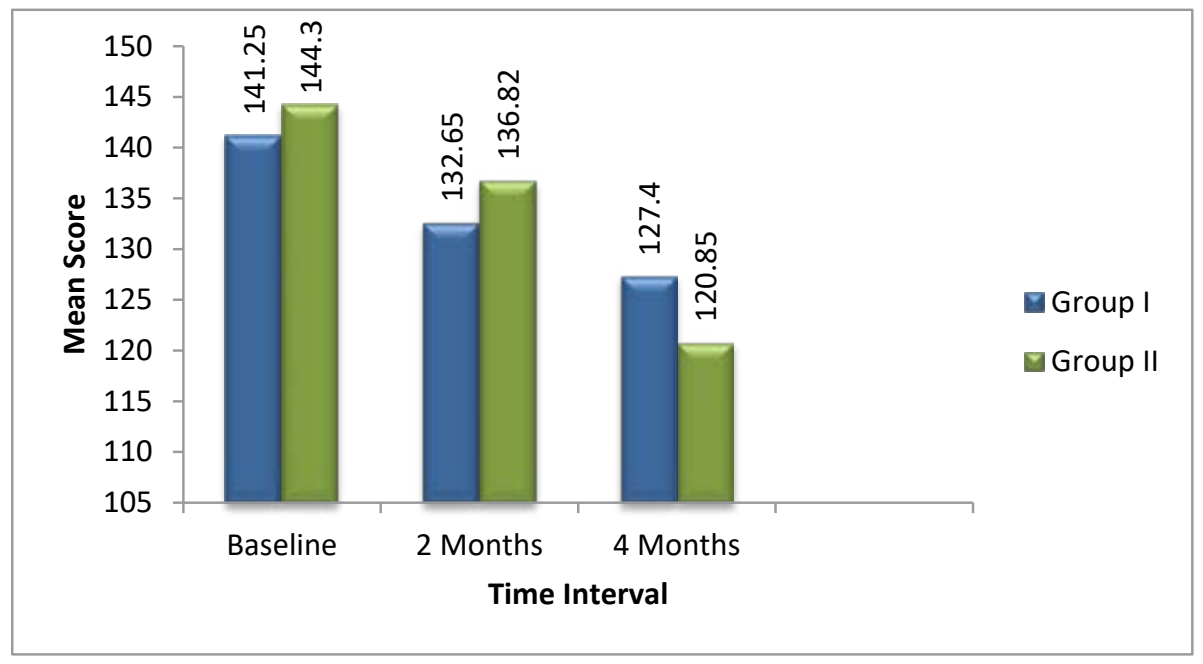

Bar Diagram Showing DBP in 2 Groups at Different Visits

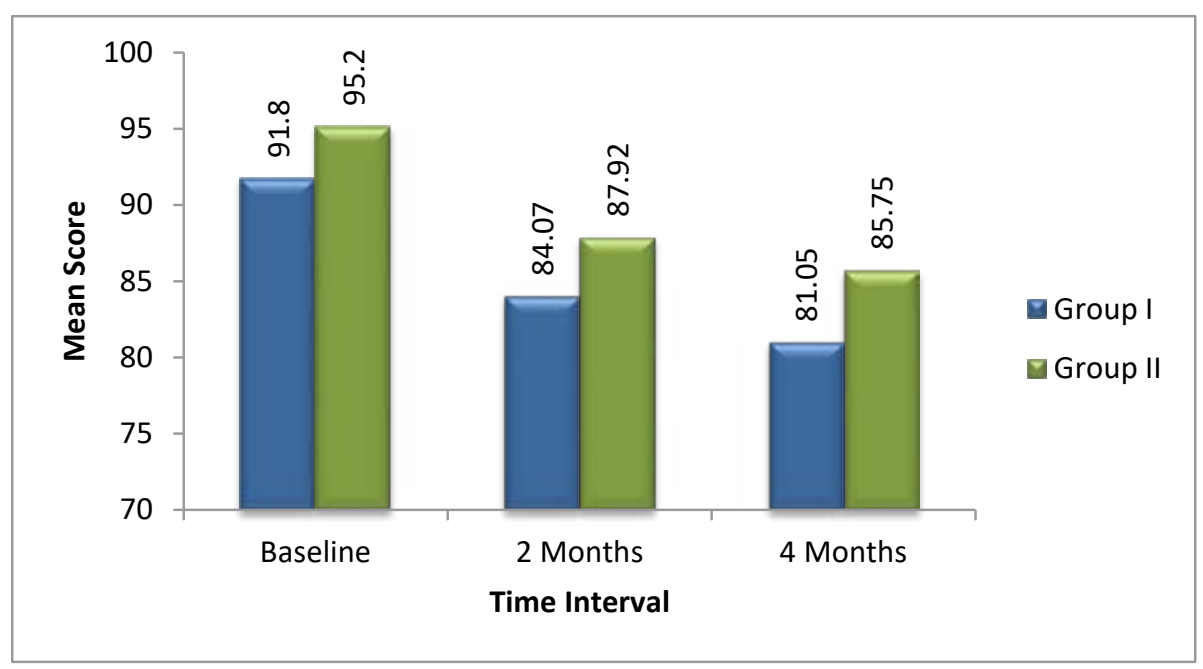


Table 3. Comparison of HR of 2 groups at different visits

\begin{tabular}{|c|c|c|c|l|c|c|}
\hline $\begin{array}{c}\text { Time } \\
\text { Interval }\end{array}$ & $\begin{array}{c}\text { Group I } \\
\text { Mean } \pm \text { SD }\end{array}$ & $\begin{array}{c}\text { Group II } \\
\text { Mean } \pm \text { SD }\end{array}$ & $\begin{array}{c}\text { Mean } \\
\text { Diff. }\end{array}$ & T & p value & Sig. \\
\hline Baseline & $77.42 \pm 11.97$ & $74.75 \pm 9.66$ & $2.67 \pm 2.31$ & 1.100 & 0.275 & NS \\
\hline 2 Months & $74.55 \pm 10.85$ & $72.40 \pm 7.35$ & $2.15 \pm 3.50$ & 1.038 & 0.303 & NS \\
\hline 4 Months & $76.30 \pm 9.15$ & $72.80 \pm 7.17$ & $3.50 \pm 1.98$ & 1.903 & 0.061 & S \\
\hline
\end{tabular}

Bar Diagram Showing HR at 2 Different Visits

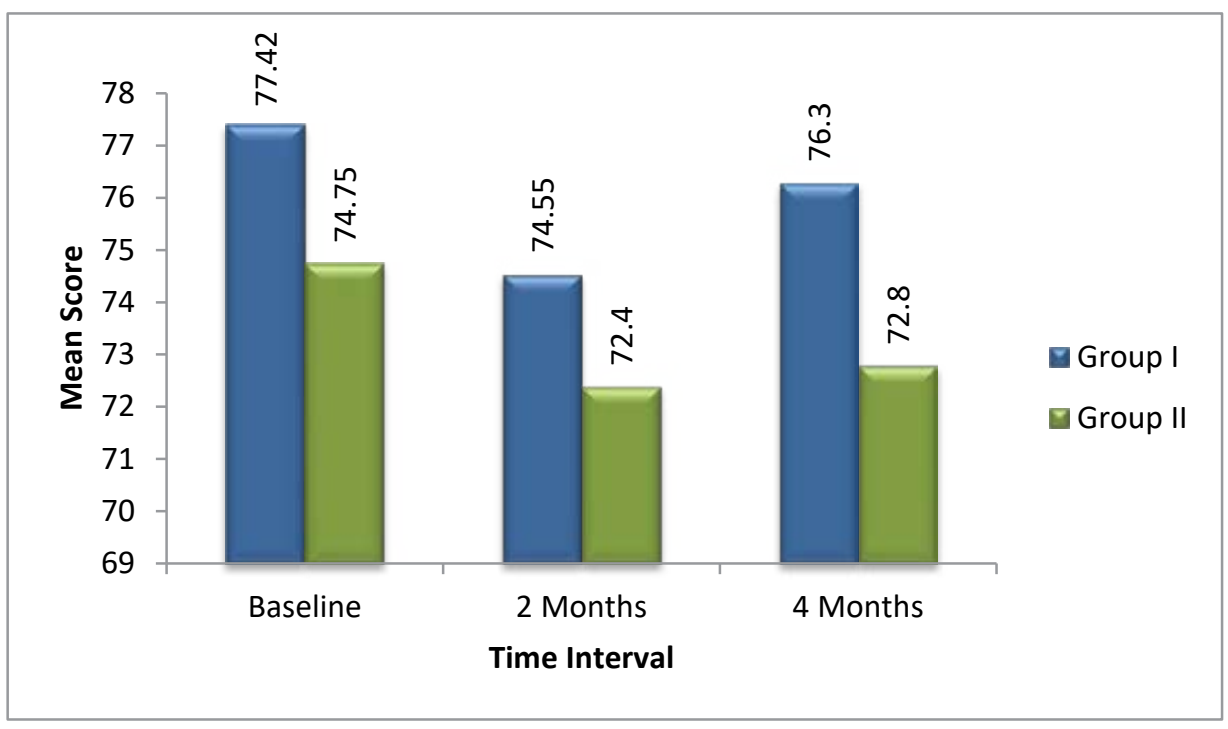

Table 4. Quality of life parameters within Metoprolol and Ramipril within group 1

\begin{tabular}{|c|c|c|c|c|c|c|c|}
\hline & & $\mathrm{N}$ & Mesn & $S D$ & $\begin{array}{l}\text { Std. } \\
\text { Error } \\
\text { Mean }\end{array}$ & t-test & $p$ value \\
\hline \multirow{2}{*}{$\begin{array}{l}\text { Physical } \\
\text { Functionin }\end{array}$} & At Bsseline & 40 & 76.37 & 25.12 & 3.97 & \multirow[b]{2}{*}{3.957} & \multirow{2}{*}{$\begin{array}{l}0.001 \\
\text { (HS) }\end{array}$} \\
\hline & At 4th Months & 40 & 89.13 & 21.36 & 3.38 & & \\
\hline \multirow{2}{*}{$\begin{array}{l}\text { Role Limitstions } \\
\text { due to Physical } \\
\text { Health }\end{array}$} & At Bsseline & 40 & 1.87 & 6.67 & 1.05 & \multirow{2}{*}{1.00} & \multirow{2}{*}{$\begin{array}{l}0.323 \\
\text { (NS) }\end{array}$} \\
\hline & At 4th Months & 40 & 4.38 & 16.88 & 2.67 & & \\
\hline \multirow{2}{*}{$\begin{array}{l}\text { Role Limitations } \\
\text { due to } \\
\text { Emotional } \\
\text { Health }\end{array}$} & At Bsseline & 40 & 0.00 & 0.00 & 0.00 & \multirow{2}{*}{1.275} & \multirow{2}{*}{$\begin{array}{l}0.210 \\
\text { (NS) }\end{array}$} \\
\hline & At 4th Months & 40 & 3.33 & 16.54 & 2.61 & & \\
\hline \multirow[t]{2}{*}{ Fatigue/Vitality } & At Baseline & 40 & 41.00 & 7.86 & 1.24 & \multirow{2}{*}{3.232} & \multirow{2}{*}{$\begin{array}{c}0.003 \\
(\mathrm{~S})\end{array}$} \\
\hline & At 4th Months & 40 & 44.25 & 7.12 & 1.13 & & \\
\hline \multirow[t]{2}{*}{$\begin{array}{l}\text { EmotionalWell } \\
\text { Being }\end{array}$} & At Bsseline & 40 & 28.70 & 5.65 & 0.89 & \multirow{2}{*}{0.313} & \multirow{2}{*}{$\begin{array}{l}0.756 \\
\text { (NS) }\end{array}$} \\
\hline & At 4th Months & 40 & 28.90 & 5.47 & 0.87 & & \\
\hline \multirow[t]{2}{*}{$\begin{array}{l}\text { Socigl } \\
\text { Functioning }\end{array}$} & At Bsseline & 40 & 49.69 & 10.40 & 1.64 & \multirow{2}{*}{0.443} & \multirow{2}{*}{$\begin{array}{l}0.660 \\
\text { (NS) }\end{array}$} \\
\hline & At 4th Months & 40 & 49.37 & 8.47 & 1.34 & & \\
\hline
\end{tabular}




\begin{tabular}{|l|l|l|r|r|r|r|r|}
\hline Pain & At Bsseline & 40 & 6.44 & 15.05 & 2.45 & \multirow{2}{*}{2.429} & $\begin{array}{c}0.020 \\
\text { (S) }\end{array}$ \\
\cline { 2 - 6 } & At 4th Months & 40 & 4.31 & 12.05 & 1.91 & & \\
\hline Genergl Health & At Bsseline & 40 & 54.10 & 8.22 & 1.30 & \multirow{2}{*}{5.283} & $\begin{array}{c}0.001 \\
\text { (HS) }\end{array}$ \\
\cline { 2 - 6 } & At 4th Months & 40 & 58.65 & 7.27 & 1.15 & \\
\hline
\end{tabular}

Bar Diagram Showing Domains of Quality of Life in Group 1

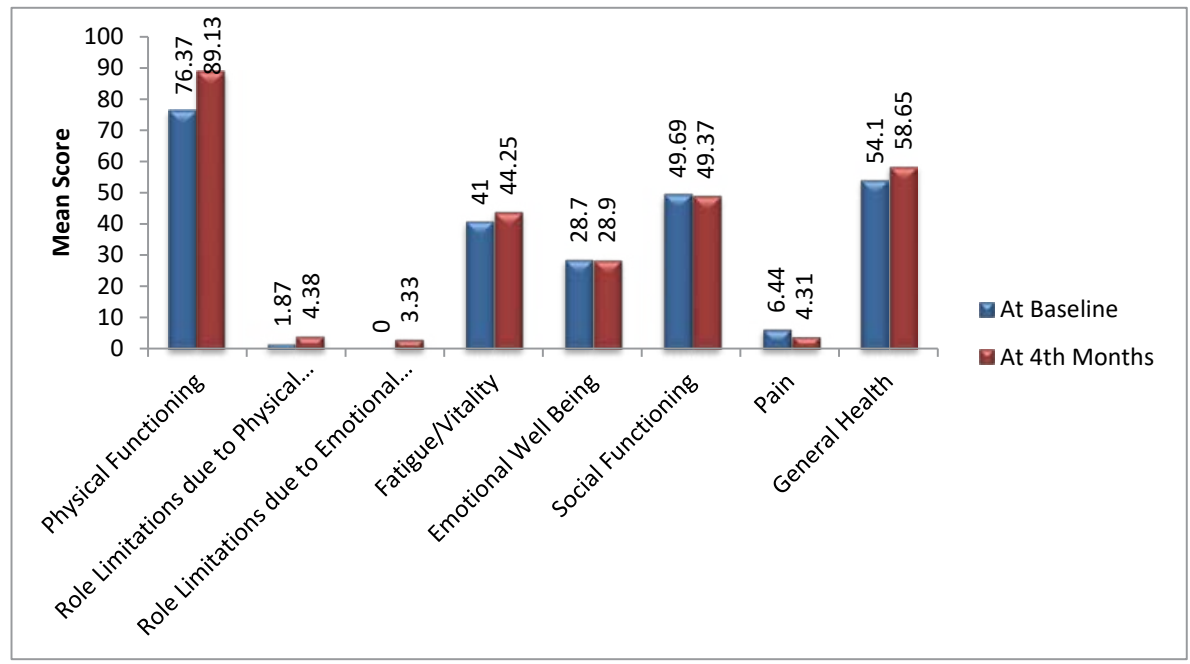

Table 5. Quality of life parameters within Metoprolol and Telmisartan within group 2

\begin{tabular}{|c|c|c|c|c|c|c|c|}
\hline & & $\mathrm{N}$ & Mesn & SD & $\begin{array}{c}\text { Std. Error } \\
\text { Mean }\end{array}$ & t-test & $\mathrm{p}$ value \\
\hline \multirow{2}{*}{$\begin{array}{l}\text { Physical } \\
\text { Functioning }\end{array}$} & At Baseline & 40 & 74.87 & 23.19 & 3.67 & \multirow[b]{2}{*}{3.452} & \multirow{2}{*}{$\begin{array}{l}0.001 \\
(\mathrm{HS})\end{array}$} \\
\hline & At 4th Months & 40 & 84.63 & 22.08 & 3.49 & & \\
\hline \multirow{2}{*}{$\begin{array}{l}\text { Role Limitstions } \\
\text { due to Physical } \\
\text { Health }\end{array}$} & At Bsseline & 40 & 10.00 & 21.78 & 3.44 & \multirow{2}{*}{1.533} & \multirow{2}{*}{$\begin{array}{l}0.133 \\
\text { (NS) }\end{array}$} \\
\hline & At 4th Months & 40 & 6.87 & 19.60 & 3.09 & & \\
\hline \multirow{2}{*}{$\begin{array}{l}\text { Role Limitations } \\
\text { due to Emotional } \\
\text { Health }\end{array}$} & At Baseline & 40 & 6.67 & 15.47 & 2.45 & \multirow{2}{*}{1.00} & \multirow{2}{*}{$\begin{array}{l}0.324 \\
\text { (NS) }\end{array}$} \\
\hline & At 4th Months & 40 & 5.00 & 14.22 & 2.24 & & \\
\hline \multirow[t]{2}{*}{ Fatigue/Vitality } & At Bsseline & 40 & 37.75 & 6.09 & 0.96 & \multirow{2}{*}{6.657} & \multirow{2}{*}{$\begin{array}{l}0.001 \\
(\mathrm{HS})\end{array}$} \\
\hline & At 4th Months & 40 & 26.75 & 10.35 & 1.64 & & \\
\hline \multirow[t]{2}{*}{$\begin{array}{l}\text { EmotionslWell } \\
\text { Being }\end{array}$} & At Bsseline & 40 & 28.29 & 6.96 & 1.10 & \multirow{2}{*}{1.684} & \multirow{2}{*}{$\begin{array}{l}0.100 \\
\text { (NS) }\end{array}$} \\
\hline & At 4th Months & 40 & 29.50 & 6.82 & 1.08 & & \\
\hline \multirow[t]{2}{*}{$\begin{array}{l}\text { Socisl } \\
\text { Functioning }\end{array}$} & At Baseline & 40 & 47.50 & 5.80 & 0.92 & \multirow{2}{*}{3.674} & \multirow{2}{*}{$\begin{array}{l}0.001 \\
\text { (HS) }\end{array}$} \\
\hline & At 4th Months & 40 & 51.25 & 5.52 & 0.87 & & \\
\hline \multirow[t]{2}{*}{ Pain } & At Bsseline & 40 & 10.50 & 21.06 & 3.33 & \multirow{2}{*}{1.740} & \multirow{2}{*}{$\begin{array}{l}0.090 \\
\text { (NS) }\end{array}$} \\
\hline & At 4th Months & 40 & 8.81 & 17.75 & 2.81 & & \\
\hline \multirow[t]{2}{*}{ General Health } & At Baseline & 40 & 50.52 & 9.86 & 1.56 & \multirow{2}{*}{5.433} & \multirow{2}{*}{$\begin{array}{c}0.001 \\
(\mathrm{~S})\end{array}$} \\
\hline & At 4th Months & 40 & 56.04 & 11.20 & 1.77 & & \\
\hline
\end{tabular}


Bar Diagram Showing Domains of Quality of Life in Group 2

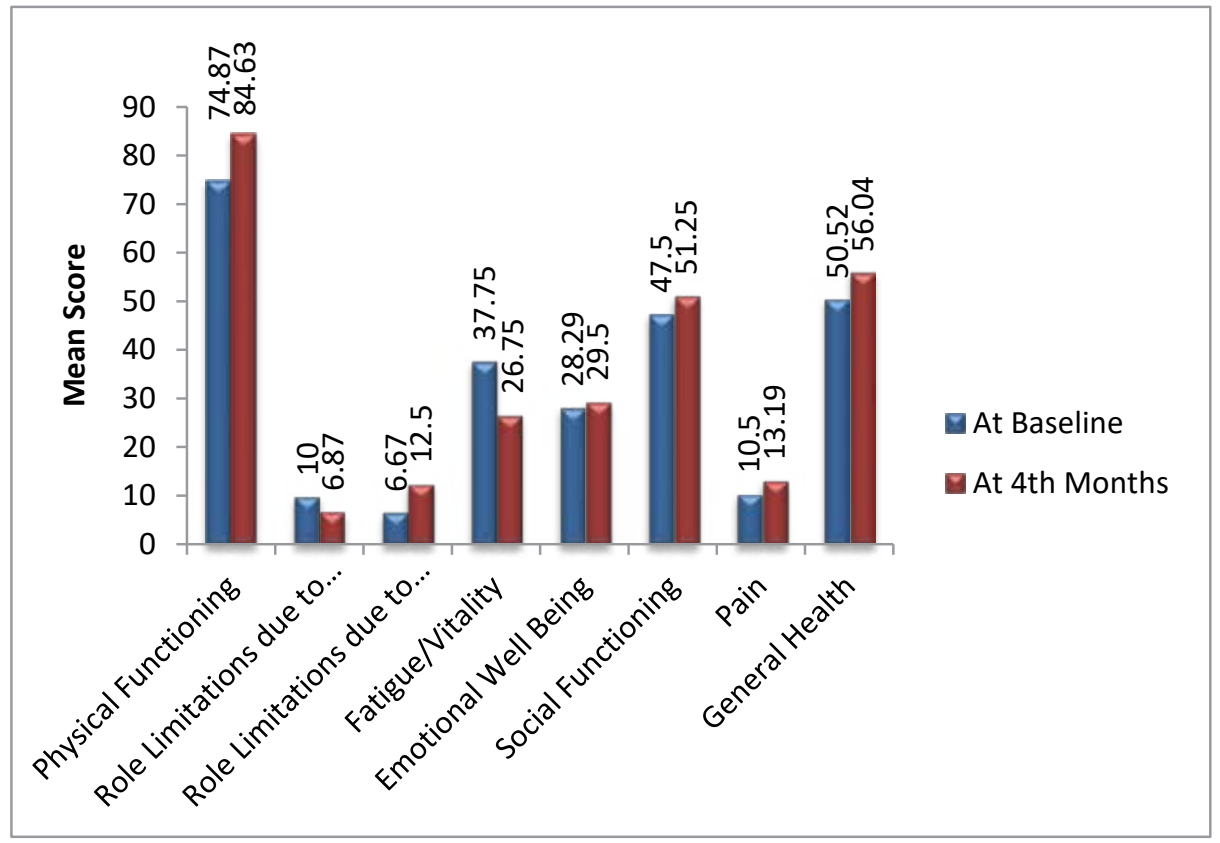

Table 6. Comparison of quality of life parameter between 2 groups at baseline

\begin{tabular}{|c|c|c|c|c|c|c|c|}
\hline At Baseline & Groups & $\mathrm{N}$ & Mean & $\mathrm{SD}$ & $\begin{array}{l}\text { Error } \\
\text { Mean }\end{array}$ & t-test & p value \\
\hline \multirow{2}{*}{$\begin{array}{l}\text { Physical } \\
\text { Functioning }\end{array}$} & Group 1 & 40 & 76.38 & 25.11 & 3.97 & \multirow[b]{2}{*}{0.278} & \multirow{2}{*}{$\begin{array}{l}0.782 \\
\text { (NS) }\end{array}$} \\
\hline & Group 2 & 40 & 74.88 & 23.19 & 3.67 & & \\
\hline \multirow{2}{*}{$\begin{array}{l}\text { Role } \\
\text { Limitations } \\
\text { due to } \\
\text { Physical } \\
\text { Health }\end{array}$} & Group 1 & 40 & 1.88 & 6.67 & 1.05 & \multirow[b]{2}{*}{2.256} & \multirow[b]{2}{*}{$\begin{array}{c}0.027 \\
\text { (S) }\end{array}$} \\
\hline & Group 2 & 40 & 10.00 & 21.78 & 3.44 & & \\
\hline \multirow{2}{*}{$\begin{array}{l}\text { Role } \\
\text { Limitations } \\
\text { due to } \\
\text { Emotional } \\
\text { Health }\end{array}$} & Group 1 & 40 & 0.00 & 0.00 & 0.00 & \multirow[b]{2}{*}{2.725} & \multirow{2}{*}{$\begin{array}{c}0.008 \\
\text { (S) }\end{array}$} \\
\hline & Group 2 & 40 & 6.67 & 15.47 & 2.45 & & \\
\hline \multirow[t]{2}{*}{ Fatigue/Nitality } & Group 1 & 40 & 41.00 & 7.86 & 1.24 & \multirow{2}{*}{2.067} & \multirow{2}{*}{$\begin{array}{c}0.042 \\
\text { (S) }\end{array}$} \\
\hline & Group 2 & 40 & 37.75 & 6.09 & 0.96 & & \\
\hline \multirow{2}{*}{$\begin{array}{l}\text { Emotional } \\
\text { Well Being }\end{array}$} & Group 1 & 4 & 28.70 & 5.65 & 0.89 & \multirow{2}{*}{0.494} & \multirow{2}{*}{$\begin{array}{l}0.623 \\
\text { (NS) }\end{array}$} \\
\hline & Group 2 & 40 & 28.00 & 6.96 & 1.10 & & \\
\hline \multirow{2}{*}{$\begin{array}{l}\text { Social } \\
\text { Functioning }\end{array}$} & Group 1 & 40 & 49.69 & 10.40 & 1.64 & \multirow{2}{*}{1.162} & \multirow{2}{*}{$\begin{array}{c}0.249 \\
\text { (NS) }\end{array}$} \\
\hline & Group 2 & 40 & 47.50 & 5.80 & 0.92 & & \\
\hline \multirow[t]{2}{*}{ Pain } & Group 1 & 40 & 6.44 & 15.50 & 2.45 & \multirow{2}{*}{0.983} & \multirow{2}{*}{$\begin{array}{c}0.329 \\
\text { (NS) }\end{array}$} \\
\hline & Group 2 & 40 & 10.50 & 21.06 & 3.33 & & \\
\hline \multirow[t]{2}{*}{$\begin{array}{l}\text { General } \\
\text { Health }\end{array}$} & Group 1 & 40 & 54.10 & 8.22 & 1.30 & \multirow{2}{*}{1.766} & \multirow{2}{*}{$\begin{array}{l}0.081 \\
\text { (NS) }\end{array}$} \\
\hline & Group 2 & 40 & 50.52 & 9.86 & 1.56 & & \\
\hline
\end{tabular}


Bar Diagram Showing Domains of Quality of Life Between 2 Groups at Baseline

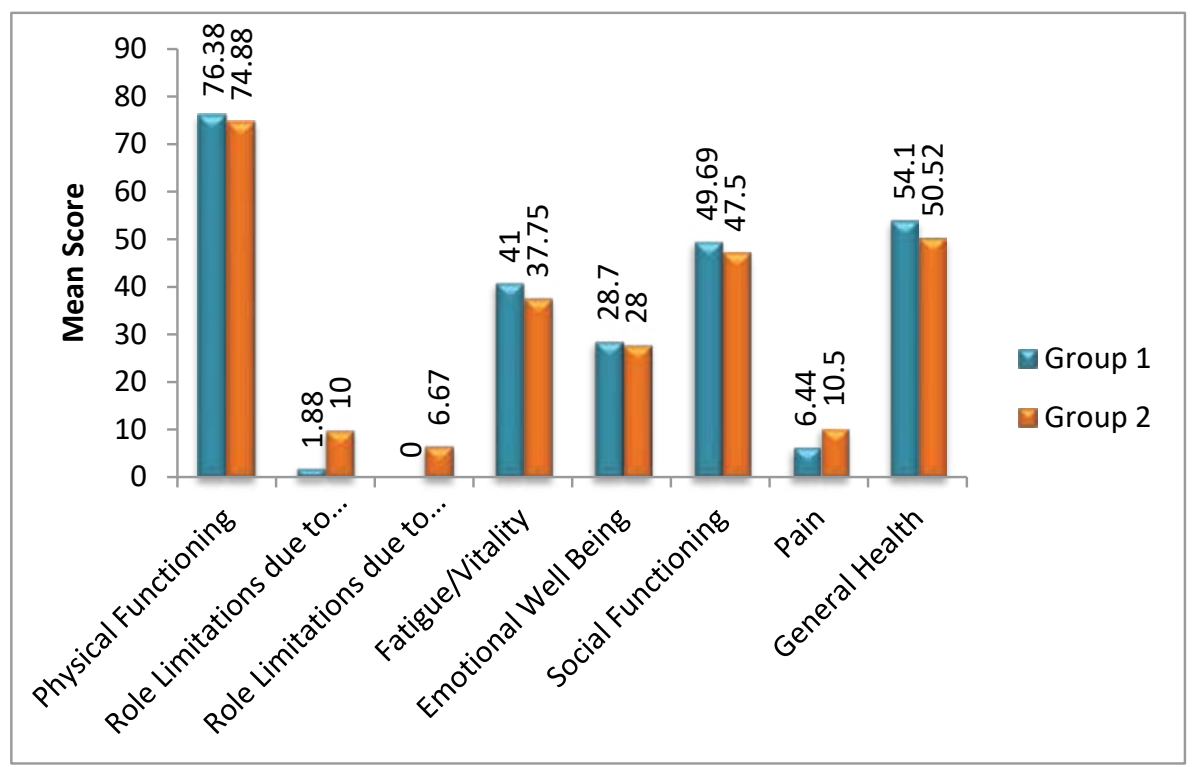

Table 7. Comparison of quality of life between 2 groups at $4^{\text {th }}$ month

\begin{tabular}{|c|c|c|c|c|c|c|c|}
\hline At 4th Months & Groups & $\mathrm{N}$ & Mean & $\mathrm{SD}$ & $\begin{array}{l}\text { Std. Error } \\
\text { Mean }\end{array}$ & t-test & $p$ value \\
\hline \multirow[t]{2}{*}{ Physical Functioning } & Group 1 & 40 & 89.13 & 21.36 & 3.38 & \multirow[b]{2}{*}{0.926} & \multirow{2}{*}{$\begin{array}{l}0.357 \\
\text { (NS) }\end{array}$} \\
\hline & Group 2 & 40 & 84.63 & 22.08 & 3.49 & & \\
\hline \multirow{2}{*}{$\begin{array}{l}\text { Role Limitations due } \\
\text { to Physical Health }\end{array}$} & Group 1 & 40 & 4.38 & 16.88 & 2.67 & \multirow[b]{2}{*}{0.611} & \multirow{2}{*}{$\begin{array}{l}0.543 \\
\text { (NS) }\end{array}$} \\
\hline & Group 2 & 40 & 6.88 & 19.60 & 3.10 & & \\
\hline \multirow{2}{*}{$\begin{array}{l}\text { Role Limitations due } \\
\text { to Emotional Health }\end{array}$} & Group 1 & 40 & 3.33 & 16.54 & 2.61 & \multirow{2}{*}{2.018} & \multirow{2}{*}{$\begin{array}{l}0.047 \\
(\mathrm{~S})\end{array}$} \\
\hline & Group 2 & 40 & 12.50 & 23.49 & 3.71 & & \\
\hline \multirow[t]{2}{*}{ Fatigue $/$ Vitality } & Group 1 & 40 & 44.25 & 7.12 & 1.13 & \multirow{2}{*}{8.810} & \multirow{2}{*}{$\begin{array}{l}0.001 \\
(\mathrm{HS})\end{array}$} \\
\hline & Group 2 & 40 & 26.75 & 10.35 & 1.64 & & \\
\hline \multirow[t]{2}{*}{ EmotionalWellBeing } & Group 1 & 40 & 28.90 & 5.47 & 0.87 & \multirow{2}{*}{0.434} & \multirow{2}{*}{$\begin{array}{c}0.665 \\
\text { (NS) }\end{array}$} \\
\hline & Group 2 & 40 & 29.50 & 6.82 & 1.08 & & \\
\hline \multirow[t]{2}{*}{ Social Functioning } & Group 1 & 40 & 49.38 & 8.47 & 1.34 & \multirow{2}{*}{1.173} & \multirow{2}{*}{$\begin{array}{l}0.244 \\
\text { (NS) }\end{array}$} \\
\hline & Group 2 & 40 & 51.25 & 5.52 & 0.87 & & \\
\hline \multirow[t]{2}{*}{ Pain } & Group 1 & 40 & 4.31 & 12.05 & 1.91 & \multirow[b]{2}{*}{2.379} & \multirow{2}{*}{$\begin{array}{l}0.020 \\
(\mathrm{~S})\end{array}$} \\
\hline & Group 2 & 40 & 13.19 & 20.29 & 3.21 & & \\
\hline \multirow[t]{2}{*}{ General Health } & Group 1 & 40 & 58.65 & 7.27 & 1.15 & \multirow{2}{*}{1.327} & \multirow{2}{*}{$\begin{array}{c}0.188 \\
\text { (NS) }\end{array}$} \\
\hline & Group 2 & 40 & 56.04 & 11.20 & 1.77 & & \\
\hline
\end{tabular}


Bar Diagram Showing Domains of Quality of Life Between 2 Groups at $4^{\text {th }}$ Month

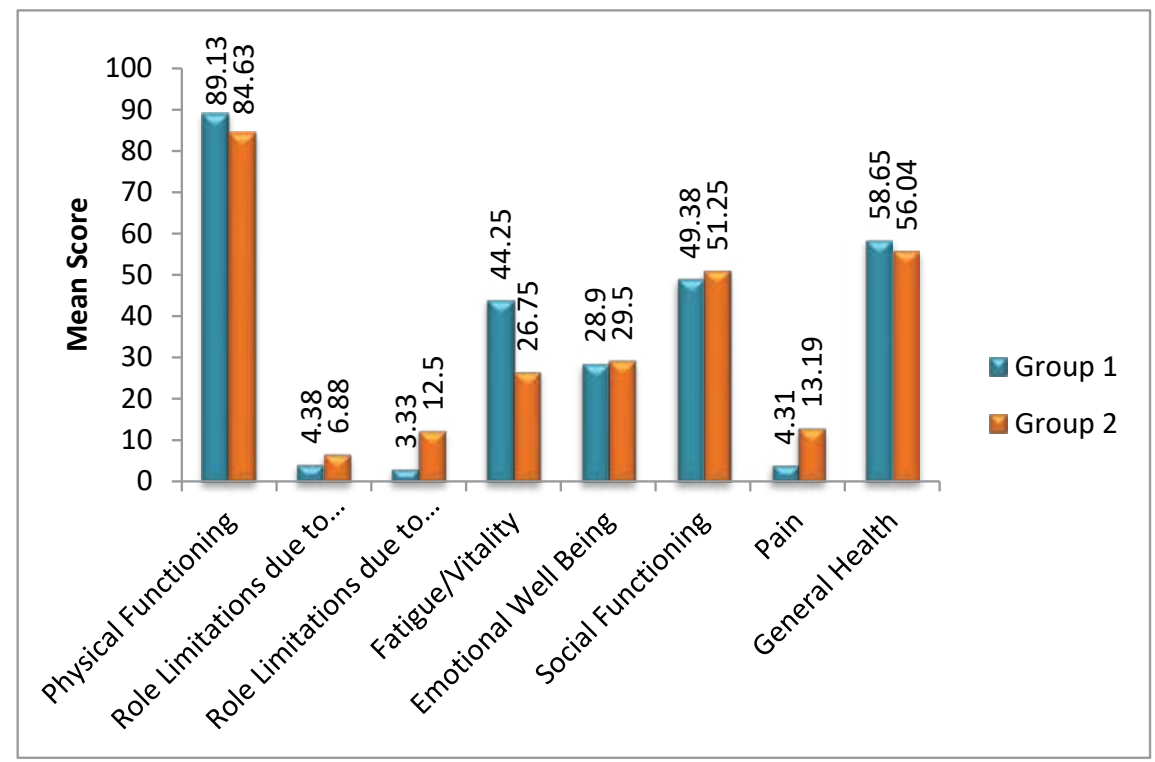

\section{Discussion}

Hypertension is a major health problem. Hypertension ranks number one amongst the non-communicable diseases. ${ }^{[10]}$

Here my study included combination therapy with two drugs to patients. The patients were prescribed Metoprolol and Ramipril in group 1 and Metoprolol and Telmisartan in group 2 in Rajindra Hospital, Patiala. Metoprolol being the common drug in two groups. Out of Telmisartan and Ramipril, Telmisartan was found to be more effective drug than Ramipril. Metoprolol, a betaadrenergic receptor antagonist was given in combination with these two separately to have an additive action on both these drugs to treat raised BP.

The fall in BP was more with Metoprolol and Telmisartan than Metoprolol and Ramipril. Similarly, there was a fall in heart rate in Metoprolol and Telmisartan than Metoprolol and Ramipril. Also, in my study I had reported no adverse effect with both the groups although adverse effects may occur with Ramipril as angioedema and cough.

In my study of Quality of life with both the groups there was a significant difference in role limitations due to emotional health and pain and there was a highly significant difference in fatigue or vitality.

MAPHY study showed the significantly lower risk for coronary events for Metoprolol as compared to diuretics. ${ }^{[1]}$
The clinicians regard ACE inhibitors and ARBs as equally effective drugs although it is not clear whether it is appropriate. ${ }^{[12]}$

A study conducted by Shahin et al., reported that ACE inhibitors improve endothelial function and are superior to beta - blockers. ${ }^{[11]}$

According to some study there was a significant change in BP and HR at the end of twelve weeks ( $\mathrm{p}<$ 0.001). When an intergroup comparison was made in Metoprolol and Telmisartan groups, there was no significant difference $(\mathrm{p}>0.05)$ at baseline but at twelve weeks the values of $\mathrm{BP}$ and $\mathrm{HR}$ showed a significant difference $(\mathrm{p}<0.05){ }^{[13]}$

The idea that the ACEI/ARB to be used as cardioprotective agents came from placebo - controlled trials in patients at high risk for cardiovascular events. ${ }^{[10]}$

From several studies Telmisartan is better in lowering blood pressure efficacy as compared with other ARBs. ${ }^{[14]}$

There were six trials were done which compared Telmisartan with Ramipril and with Telmisartan there was a greater SBP reduction. ${ }^{[15]}$

Studies regarding HRQOL are mostly conflicting with some studies show worse HRQOL but here the mechanism for the low HRQOL is not known and some studies show no impact of hypertension in some or all domains. ${ }^{[16]}$

Recent study shows that by using ACEI or ARBs had high scores on HRQOL assessment scale. ${ }^{[17]}$ 


\section{Conclusion}

Following conclusion was drawn from my study.

Although hypertension is a world-wide problem, it can be treated with single pill combination however if the desired BP lowering does not occur then double or triple pill combination can be used. The blood pressure and heart rate decreased more with Metoprolol and Telmisartan combination as compared with Metoprolol and Ramipril also quality of life improved better with Metoprolol and Telmisartan in role limitations due to emotional health and pain and majority in parameter of fatigue or vitality. Quality of life was assessed with SF-36 questionnaire.

\section{References}

1. Benndorf RA, et al. Pleiotropic effects of telmisartan: Still more to come? J Hypertension. 2008; 26(5):854-6. https:// doi.org/10.1097/HJH.0b013e3282f76481 PMid:18398324

2. Stafylas PC, Sarafidis PA. Carvedilol in hypertension treatment. Vasc Health Risk Manag. 2008 Feb; 4(1):23-30. https://doi.org/10.2147/vhrm.2008.04.01.23 PMid:18629377 PMCid:PMC2464772

3. Chobanian AV, Bakris GL, Black HR, Cushman WC, Green LA, Izzo JL, et al. Seventh Report of the joint national committee on prevention, detection, evaluation, and treatment of high blood pressure: The JNC report. JAMA. 2003; 289(19):2560-72. https://doi.org/10.1001/ jama.289.19.2560 PMid:12748199

4. Gupta R. Trends in hypertension epidemiology in India. J Hum Hypertens. 2004 Feb; 18(2):73-8. https://doi. org/10.1038/sj.jhh.1001633 PMid:14730320

5. Chrysant SG, Chyrysant GS, Dimas B. Current and future status of beta- blockers in the treatment of hypertension. Clinical Cardiology. 2008 Jun; 31(6):249-52. https://doi. org/10.1002/clc.20249 PMid:18543303

6. Saseen JJ, MacLaughlin. Hypetension. DiPiro JT, Talbert RL, Yee GC, Matzke GR, Wells BG, Posey LM, editors. Pharmacotherapy: A pathophysiologic approach. 9th ed. New York: McGraw-Hill Medical; 2014.

7. CDC: High blood pressure. Centers for Disease Control and Prevention; 2015. Available from: http://www.cdc.gov/ bloodpressure/index.htm

8. James PA, Oparil S, Carter BL, Cushman WC, DennisonHimmelfarb C, Handler K, Lackland DT, LeFevre M, MacKenzie TD, Ogedegbe O, Smith SC, Svetkey LP, Taler
SJ, Townsend RR, Wright J, Narva AS, Ortiz E. Evidence based guideline for the management of high blood pressure in adults: report from the panel members appointed to the Eighth Joint National Committee (JNC 8). JAMA. 2014; 311(5):507-20. Available from: http://jama.jamanetwork.com/article.aspx?articleid=1791497 https://doi. org/10.1001/jama.2013.284427 PMid:24352797

9. Friedrich MG, Dahlof B, Sechtem U, Unger T, Knecht M. Telmisartan Effectiveness on Left Ventricular Mass Reduction (TELMAR) as assessed by mabnetic resonance imaging in patients with mild-to-moderate hypertension- a prospective, randomized, double- blind comparison of telmisartan with metoprolol over a period of six months- rationale and study design. Journal of the Renin-Angiotensin-Aldosterone System. 2003 Dec; 4(4):234-43. https://doi.org/10.3317/jraas.2003.038 PMid:14689371

10. Ong HT, FRCP, Rozina G, FRCP. Selecting antihypertensive medication in patients with essential hypertension in Malaysia. Med J Malaysia. 2009; 64(1):2009 Mar.

11. Tomiyama H, Yamashina A. Beta- blockers in the management of hypertension and/or chronic kidney disease. International Journal of Hypertension. 2014; 1-7. https://doi.org/10.1155/2014/919256 PMid:24672712 PMCid:PMC3941231

12. Matchar DB, McCrory DC, Orlando LA, Patel MR, Patel UD, Patwardhan MB, et al. Systematic review: Comparative effectiveness of angiotensin- converting enzyme inhibitors and angiotensin II receptor blockers for treating essential hypertension. Ann Intern Med. 2008; 148:16-29. https:// doi.org/10.7326/0003-4819-148-1-200801010-00189 PMid:17984484

13. Agarwal A, Chhabra MK, Walia R, Gupta PD. Comparative evaluation of Metoprolol and Telmisartan in hypertensive patients. 2014 Jul; 3(2): 403-10. Available from: www. Ijmds.org

14. Kumar P, Kapoor AK, Singh HK, Kulshrestha M, Randomized. Interventional, prospective, comparative study to evaluate the antihypertensive efficacy and tolerability of Ramipril versus Telmisartan in stage 1 hypertensive patients with diabetes mellitus. Internet Journal of Medical Update. 2015 Jan; 10(1):15-25. https://doi.org/10.4314/ ijmu.v10i1.4

15. Zou Z, Xi G-L, Yuan HB, Zhu Q-F, Shi X-Y. Telmisartan versus Angiotensin converting enzyme inhibitors in the treatment of hypertension: a meta-analysis of randomized controlled trials. Journals of Human Hypertension. 2008; 23(5):1-11. 
16. Soni RK, Porter AC, Lash JP, Unruh ML. Health-related quality of life in hypertension, chronic kidney disease and coexistent chronic health conditions. Adv Chronic Kidney Dis. 2010 Jul; 17(4):e17-26. https://doi.org/10.1053/j. ackd.2010.04.002 PMid:20610351 PMCid:PMC2901238
17. Shanableh S, Abdulkarem A, Shamssain M, Sarhan F. Quality of life of hypertensive patients on different types of antihypertensive medications. IOSR Journal of Pharmacy. 2014 May; 4(5):23-8. https://doi.org/10.9790/30130405023028

How to cite this article: Arora S., Sehgal V. K., Singh J. and Singh H. Comparative Study to Evaluate Efficacy, Safety and Quality of Life of Metoprolol and Telmisartan Versus Metoprolol and Ramipril In Patients of Hypertension. Int. J. Med. Dent. Sci. 2019; 8(2):1728-1738. 